\title{
Discrete Velocity Models for Polyatomic Molecules Without Nonphysical Collision Invariants
}

\author{
Niclas Bernhoff ${ }^{1}$ (DD
}

Received: 2 November 2017 / Accepted: 11 May 2018 / Published online: 19 May 2018

(C) The Author(s) 2018

\begin{abstract}
An important aspect of constructing discrete velocity models (DVMs) for the Boltzmann equation is to obtain the right number of collision invariants. Unlike for the Boltzmann equation, for DVMs there can appear extra collision invariants, so called spurious collision invariants, in plus to the physical ones. A DVM with only physical collision invariants, and hence, without spurious ones, is called normal. The construction of such normal DVMs has been studied a lot in the literature for single species, but also for binary mixtures and recently extensively for multicomponent mixtures. In this paper, we address ways of constructing normal DVMs for polyatomic molecules (here represented by that each molecule has an internal energy, to account for non-translational energies, which can change during collisions), under the assumption that the set of allowed internal energies are finite. We present general algorithms for constructing such models, but we also give concrete examples of such constructions. This approach can also be combined with similar constructions of multicomponent mixtures to obtain multicomponent mixtures with polyatomic molecules, which is also briefly outlined. Then also, chemical reactions can be added.
\end{abstract}

Keywords Boltzmann equation · Discrete velocity models · Collision invariants · Polyatomic molecules

Mathematics Subject Classification $82 \mathrm{C} 40 \cdot 35 \mathrm{Q} 20 \cdot 76 \mathrm{P} 05$

\section{Introduction}

We consider the Boltzmann equation for polyatomic molecules [19,25,29], here represented by that each molecule has an internal energy that can change during collisions. In particular, we study discrete velocity models (DVMs), i.e., we assume that the velocity variable only

$\triangle$ Niclas Bernhoff

niclas.bernhoff@kau.se

1 Department of Mathematics and Computer Science, Karlstad University, 65188 Karlstad, Sweden 
can take a finite number of different given (vector) values. The Boltzmann equation can be approximated by DVMs up to any order [17,20,26,32], and these discrete approximations can be used for numerical methods, e.g., see [20,31] and references therein. However, in the construction of DVMs there is a classical question of having the correct number of collision invariants [27]. Unlike in the continuous case, there can be additional collision invariants to the physical ones; mass momentum, and energy, for DVMs. DVMs, without additional collision invariants, for which the collision invariants are linearly independent, are called normal. The construction of normal DVMs for single species as well as for binary mixtures has been well studied, see for example [15,16,18,23,24,35-37], and recently also for multicomponent mixtures [10]. We like to point out, that in [16] main ideas of a general approach to the construction of normal discrete kinetic models, including a brief discussion on the application to DVMs for inelastic collisions, is presented. Still in particular cases, more detailed studies are needed. We consider here the problem of constructing DVMs for single species of polyatomic molecules with the right number of collision invariants and outline the extension to DVMs for mixtures of polyatomic molecules (cf. [7]). We like to stress that we thereby also can handle the important case of mixtures of several monatomic and polyatomic gases that, e.g., is of importance during the reentry of space shuttles in the upper atmosphere [1].

Unlike for single species, each polyatomic molecule has an internal energy, to account for non-translational energies. We assume that the set of allowed internal energies is finite and add to each internal energy a finite set of allowed velocities. In this way we obtain pairs of allowed velocities and internal energies, cf. [21], where two pairs can have the same velocity, but different internal energies, or vice versa. In correspondence with the cases of binary mixtures [16] and multicomponent mixtures [10] we introduce the concepts of semisupernormal and supernormal DVMs for polyatomic molecules. We present algorithms for constructing such DVMs and give some concrete examples of such constructions. We also prove that for any given (finite number of) multiples of an internal "basic" energy we can construct a supernormal DVM. Our constructed DVMs can always be extended to larger DVMs by the method of one-extensions [14,15,35]. A one-extension is obtained in the following way: if three out of four velocities, involved in a possible (i.e., such that the physical quantities are conserved) collision, already are in an existing normal DVM, one adds also the fourth velocity to the DVM; in this way a new normal DVM, with one more velocity, a oneextension, is obtained. We like to stress that it is always possible to extend our constructed DVMs to DVMs symmetric with respect to the axes by the method of one-extensions.

Another important issue is the one of approximating the full Boltzmann equation by DVMs, which have been addressed for single species of polyatomic molecules in e.g., [21] and for mixtures with polyatomic molecules in e.g., [28]. For simulations, it is important to have the right number of collision invariants. Our results concerning the correct number of collision invariants are independent of the modeling of the collision coefficients as long as the collision coefficients for a maximal set of linearly independent collisions (i.e., collisions that cannot be obtained by combining the other collisions, including corresponding inverse collisions, in the set) are nonzero, which will be implicitly assumed below. In [21] it is proven, that for both of the (families of) models considered there, the collision invariants are the physical ones, and only those. However, our results are more general and can be applied to a much larger family of DVMs, including those in [21]. We want to stress that our intention is not at all to discuss different implementations, but instead to make a more careful investigation of normality for DVMs in the case of polyatomic molecules.

The construction of the DVMs is made such that for half-space problems [3], as the linearized Milne and Kramers problems [2], but also nonlinear ones [34], one obtains similar 
structures as for the classical discrete Boltzmann equation for one species $[4,5,8,13]$, and can extend corresponding results, cf. [10] for multicomponent mixtures. The same is also true for the analytically difficult problem of existence of shock profiles [22,30], where one also obtains a similar structure as for the classical discrete Boltzmann equation for one species [9] and can extend corresponding results, see [7].

The remaining part of the paper is organized as follows. Section 2 concerns DVMs for polyatomic molecules; the concepts of semi-supernormal and supernormal DVMs, respectively, for polyatomic molecules are introduced in Sect. 2.1, and algorithms for constructing such models is presented in Sect. 2.2. Section 3 concerns concrete examples of such DVMs. It is also proven that for any given (finite number of) multiples of an internal "basic" energy we can construct a supernormal DVM in Sect. 3. In Sect. 4 we outline the extension to multicomponent mixtures with polyatomic molecules and state some corresponding results.

\section{DVMs for Polyatomic Molecules}

We consider a single species with polyatomic molecules, in the sense that each particle has an associated internal energy $[12,19,29]$. The total number of possible internal energies is assumed to be finite, i.e., there are $s$ different internal energies $E^{1}, \ldots, E^{s}$ that can be associated with the particles, which either can be considered as that there only is a finite number of different (internal) energy states or that one (in some way) have modelled a continuous internal energy variable by discretizing it, cf. [21]. We model the Boltzmann equation by discretizing the continuous velocity variable, i.e., we assume that the velocity only can take a finite number of different vector values. In order to do the discretization, we fix a set of velocity vectors $V_{i}=\left\{\xi_{1}^{i}, \ldots, \xi_{n_{i}}^{i}\right\} \subset \mathbb{R}^{d}$ (in applications $d=2,3$ ) for each of the internal energies $E^{i}$. Now there are $n=n_{1}+\cdots+n_{s}$ different pairs, being composed of a velocity vector and an internal energy, contained in the set

$$
\begin{aligned}
\mathscr{V} & =\left\{\left(\xi_{1}^{1}, E^{1}\right), \ldots,\left(\xi_{n_{1}}^{1}, E^{1}\right), \ldots,\left(\xi_{1}^{s}, E^{s}\right), \ldots,\left(\xi_{n_{s}}^{s}, E^{s}\right)\right\} \\
& =\left\{\left(\mathbf{v}_{1}, E_{1}\right), \ldots,\left(\mathbf{v}_{n}, E_{n}\right)\right\}, \quad n=n_{1}+\cdots+n_{s} .
\end{aligned}
$$

Note that the same velocity can appear many times in the set, while the pairs are unique. An important question is how to choose the sets of velocities, in such a way that there will be no extra, so called spurious, collision invariants, in plus to the physical ones. Our main concern is how the velocity sets can be constructed, such that the resulting DVM will be normal, i.e., without spurious collision invariants. Explicit implementations will not be considered here, but we refer to e.g., $[21,28]$ for some interesting examples. It would also be of interest to see if the results in [20] could be extended to include also (mixtures and/or) polyatomic molecules.

The general DVM, or the discrete Boltzmann equation, for a single species with polyatomic molecules reads

$$
\frac{\partial f_{i}}{\partial t}+\mathbf{v}_{i} \cdot \nabla_{\mathbf{x}} f_{i}=Q_{i}(f, f), \quad i=1, \ldots, n,
$$

where $f_{i}=f_{i}(\mathbf{x}, t)=f\left(\mathbf{x}, t, \mathbf{v}_{i}, E_{i}\right)$ for $i=1, \ldots, n$, and $f=f(\mathbf{x}, t, \xi, E)$ represents the microscopic density of particles with internal energy $E$ and velocity $\xi$ at time $t \in \mathbb{R}_{+}$ and position $\mathbf{x} \in \mathbb{R}^{d}$. 
For a function $h=h(\xi, E)$ (possibly depending on more variables than $\xi$ and $E$ ), we identify $h$ with its restrictions to the pairs $\left(\mathbf{v}_{i}, E_{i}\right) \in \mathscr{V}$, i.e.,

$$
h=\left(h_{1}, \ldots, h_{n}\right), \quad h_{i}=h\left(\mathbf{v}_{i}, E_{i}\right), \quad i=1, \ldots, n .
$$

Then $f=\left(f_{1}, \ldots, f_{n}\right)$ in Eq. (1).

The collision operators $Q_{i}(f, f)$ in (1) are given by

$$
Q_{i}(f, f)=\sum_{j, k, l=1}^{n} \Gamma_{i j}^{k l}\left(f_{k} f_{l}-f_{i} f_{j}\right), \quad i=1, \ldots, n,
$$

where we assume that the collision coefficients satisfy the symmetry relations

$$
\Gamma_{i j}^{k l}=\Gamma_{j i}^{k l}=\Gamma_{k l}^{i j} \geq 0
$$

with equality unless we have conservation of momentum and total energy

$$
\begin{aligned}
\mathbf{v}_{i}+\mathbf{v}_{j} & =\mathbf{v}_{k}+\mathbf{v}_{l}, \\
\frac{m\left|\mathbf{v}_{i}\right|^{2}}{2}+\frac{m\left|\mathbf{v}_{j}\right|^{2}}{2}+E_{i}+E_{j} & =\frac{m\left|\mathbf{v}_{k}\right|^{2}}{2}+\frac{m\left|\mathbf{v}_{l}\right|^{2}}{2}+E_{k}+E_{l} .
\end{aligned}
$$

Furthermore, we assume that $\Gamma_{i j}^{k l}=0$ if

$$
\mathbf{v}_{i}=\mathbf{v}_{j} \text { or } \mathbf{v}_{k}=\mathbf{v}_{l} .
$$

This assumption was not considered in $[7,8]$. However, even if particles with the same velocity, by obvious reasons, will never collide in reality (neither we assume molecules to clog together during a collision), such reactions can still appear as combinations of collisions involving other velocities (belonging to the considered velocity sets, or not). Moreover, they can also be chosen as a represent for collisions of molecules with velocities close to that "same" velocity (however, they might still be rare enough to neglect). Therefore, in principle, it would be possible to allow also these collisions as in $[7,8]$, even if we do not choose to do it here. Anyway, these reactions might still appear implicitly as combinations of allowed collisions.

To obtain the symmetry relations (3) we may need to scale the distribution functions first (cf. [19])

$$
f_{r}^{\prime}=\frac{f_{r}}{g_{i}} \text { if } E_{r}=E^{i}, \quad r=1, \ldots, n, \text { for some numbers } g_{1}, \ldots, g_{s} .
$$

After a possible scaling (6) of the distribution functions, the symmetry relations (3) are a natural assumption, assuming a convenient reciprocity relation, see, e.g., [25, p. 9]. We like to point out that the symmetry relations (3) are fulfilled for the proposed models in [21] (with $g_{1}^{j}=\left(E_{1}^{j}\right)^{\delta / 2-1}$, where $\delta$ is the number of internal degrees of freedom).

A collision is obtained by the exchange of velocities and internal energies

$$
\left\{\left(\mathbf{v}_{i}, E_{i}\right),\left(\mathbf{v}_{j}, E_{j}\right)\right\} \leftrightarrows\left\{\left(\mathbf{v}_{k}, E_{k}\right),\left(\mathbf{v}_{l}, E_{l}\right)\right\}
$$

and can occur if and only if $\Gamma_{i j}^{k l} \neq 0$. Geometrically, the collision obtained by (7) is, as in the case of single species, represented by a rectangle in $\mathbb{R}^{d}$, if $\left\{E_{i}, E_{j}\right\}=\left\{E_{k}, E_{l}\right\}$, with the corners in $\left\{\mathbf{v}_{i}, \mathbf{v}_{j}, \mathbf{v}_{k}, \mathbf{v}_{l}\right\}$, where $\mathbf{v}_{i}$ and $\mathbf{v}_{j}$ (and therefore, also $\mathbf{v}_{k}$ and $\mathbf{v}_{l}$ ) are diagonal corners. In general, the collision obtained by (7) is geometrically represented by a 
parallelogram in $\mathbb{R}^{d}$ [16], with the corners in $\left\{\mathbf{v}_{i}, \mathbf{v}_{j}, \mathbf{v}_{k}, \mathbf{v}_{l}\right\}$, where $\mathbf{v}_{i}$ and $\mathbf{v}_{j}$ (and therefore, also $\mathbf{v}_{k}$ and $\mathbf{v}_{l}$ ) are diagonal corners, such that

$$
m\left(\mathbf{v}_{k} \cdot \mathbf{v}_{l}-\mathbf{v}_{i} \cdot \mathbf{v}_{j}\right)=E_{k}+E_{l}-E_{i}-E_{j} .
$$

Note that the parallelogram is allowed to be degenerate, in the sense that it has no hight (or area), i.e., all corners of the parallelogram lie on the same line.

A function $\phi=\phi(\mathbf{v})$, is a (global) collision invariant if and only if

$$
\phi_{i}+\phi_{j}=\phi_{k}+\phi_{l}
$$

for all indices such that $\Gamma_{i j}^{k l} \neq 0$. We note that in general a collision invariant can also depend on time and position. However, that dependence is of no importance for our studies here and therefore, we will below assume that all collision invariants are global, i.e., that they are independent of time and position. The vector space of collision invariants contains the trivial collision invariants (or physical collision invariants)

$$
1, m v^{1}, \ldots, m v^{d}, m|\mathbf{v}|^{2}+2 E,
$$

where $v^{i}=\left(v_{1}^{i}, \ldots, v_{n}^{i}\right)$ (here we denote $\left.\mathbf{v}_{j}=\left(v_{j}^{1}, \ldots, v_{j}^{d}\right)\right),|\mathbf{v}|^{2}=\left(\left|\mathbf{v}_{1}\right|^{2}, \ldots,\left|\mathbf{v}_{n}\right|^{2}\right)$, and $E=E(\mathbf{v})=\left(E_{1}, \ldots, E_{n}\right)$.

In the continuous case, these are the only collision invariants. However, for DVMs there can also be extra, so called spurious, collision invariants. DVMs without spurious collision invariants are called normal, if the $d+2$ collision invariants (8) are linearly independent. A DVM such that the collision invariants (8) are linearly dependent are called degenerate, and otherwise non-degenerate. Methods of construction of normal DVMs for monatomic single species and mixtures can be found in e.g., $[10,15,16]$. We again stress that the collision invariants include, and for normal models are restricted to

$$
\phi=\left(\phi_{1}, \ldots, \phi_{n}\right), \quad \phi_{i}=a+m \mathbf{b} \cdot \mathbf{v}_{i}+c\left(m\left|\mathbf{v}_{i}\right|^{2}+2 E_{i}\right)
$$

for some constant $a, c \in \mathbb{R}, \mathbf{b} \in \mathbb{R}^{d}$. Our main interest in this work is the construction of such normal models.

The stationary points are Maxwellian distributions (or just Maxwellians) of the form

$$
M=e^{\phi},
$$

where (for normal models) $\phi$ is given by Eq. (9).

Note also that under the assumptions above we will have an $H$-theorem as usual, cf. [8].

\subsection{Supernormal DVMs for Polyatomic Molecules}

For DVMs for polyatomic molecules, one can, as in the case of DVMs for mixtures cf. [10,16] have different kinds of normality. Similarly as in the case of mixtures in [10], we introduce different kinds or levels of normality. We start with the usual definition of normality.

\section{Definition 1 A DVM}

$$
\left\{\left\{\mathrm{V}_{1}, E^{1}\right\}, \ldots,\left\{\mathrm{V}_{s}, E^{s}\right\}\right\}
$$

with internal energies $\left\{E^{1}, \ldots, E^{s}\right\}$, is called normal if it is non-degenerate and has exactly $d+2$ linearly independent collision invariants. 
Note that for normal DVMs, the $d+2$ linearly independent collision invariants in Definition 1 will be linear combinations of the $d+2$ trivial collision invariants (8).

A drawback with Definition 1 is that if we look separately on the restriction to a specific energy level, the reduced model does not have to be normal. Therefore, we extend the definition above.

Definition 2 A DVM (11), with internal energies $\left\{E^{1}, \ldots, E^{s}\right\}$, is called semi-supernormal if it is normal and the restriction to each velocity set $\mathrm{V}_{i}, 1 \leq i \leq s$, is a normal DVM.

However, still if we consider subsets of energy levels, the restrictions to those energy levels do not have to be normal. Therefore, we make a further extension.

Definition 3 A DVM (11), with internal energies $\left\{E^{1}, \ldots, E^{s}\right\}$, is called supernormal if the restriction to each collection

$$
\left\{\left\{\mathrm{V}_{r_{1}}, E^{r_{1}}\right\}, \ldots,\left\{\mathrm{V}_{r_{i}}, E^{r_{i}}\right\}\right\} \subseteq\left\{\left\{\mathrm{V}_{1}, E^{1}\right\}, \ldots,\left\{\mathrm{V}_{s}, E^{s}\right\}\right\}, \quad 1 \leq i \leq s,
$$

is a normal DVM.

Depending on what we are interested to study we can be satisfied with different levels of normality, where normal is the lowest level and supernormal the highest one (including the other ones).

As we construct semi-supernormal DVMs we can be helped by the following theorem.

Theorem 1 A DVM (11), with internal energies $\left\{E^{1}, \ldots, E^{s}\right\}$, is semi-supernormal if, for each $2 \leq j \leq s$ there exists $1 \leq i<j \leq s$, such that the restriction to the pair $\left\{\left\{\mathrm{V}_{i}, E^{i}\right\},\left\{\mathrm{V}_{j}, E^{j}\right\}\right\}$ is a supernormal DVM.

Proof The restriction to each velocity set $\mathrm{V}_{i}=\left\{\xi_{1}^{i}, \ldots, \xi_{n_{i}}^{i}\right\}, 1 \leq i \leq s$, is normal by the supernormality of $\left\{\left\{\mathrm{V}_{i}, E^{i}\right\},\left\{\mathrm{V}_{j}, E^{j}\right\}\right\}$. Hence, the collision invariants will be of the form $\phi=\left(\phi^{1}, \ldots, \phi^{s}\right)$, where $\phi_{j}^{i}=a_{i}+m \mathbf{b}^{i} \cdot \xi_{j}^{i}+c_{i}\left(m\left|\xi_{j}^{i}\right|^{2}+2 E^{i}\right)$ for $1 \leq j \leq n_{i}$ and $1 \leq i \leq s$.

Denote $a_{1}=a, \mathbf{b}^{\mathbf{1}}=\mathbf{b}$, and $c_{1}=c$. Assume that $a_{j-1}=a_{j-2}=\cdots=a_{1}=a$, $\mathbf{b}^{j-1}=\mathbf{b}^{j-2}=\cdots=\mathbf{b}^{1}=\mathbf{b}$, and $c_{j-1}=c_{j-2}=\cdots=c_{1}=c$ for some $2 \leq j \leq s$. Then there exists $1 \leq i \leq j-1$, such that the restriction to the pair $\left\{\left\{\mathrm{V}_{i}, E^{i}\right\},\left\{\mathrm{V}_{j}, E^{j}\right\}\right\}$ is normal and therefore $a_{j}=a_{i}=a, \mathbf{b}^{j}=\mathbf{b}^{i}=\mathbf{b}$, and $c_{j}=c_{i}=c$. Hence, the collision invariants will be of the form $\phi=\left(\phi^{1}, \ldots, \phi^{s_{s}}\right)$, where $\phi_{j}^{i}=a+m \mathbf{b} \cdot \xi_{j}^{i}+c\left(m\left|\xi_{j}^{i}\right|^{2}+2 E^{i}\right)$ for $1 \leq j \leq n_{i}$ and $1 \leq i \leq s$.

For constructing supernormal DVMs (or checking if existing DVMs are supernormal), the following theorem can be useful.

Theorem 2 A DVM (11), with internal energies $\left\{E_{1}, \ldots, E_{s}\right\}$, is supernormal if and only if the restriction to each pair

$$
\left\{\left\{\mathrm{V}_{i}, E^{i}\right\},\left\{\mathrm{V}_{j}, E^{j}\right\}\right\}, \quad 1 \leq i<j \leq s,
$$

of velocity sets is a supernormal DVM.

Proof The theorem follows directly from the definition of supernormal DVMs and Theorem 1. 


\subsection{Algorithms for Construction of Semi-supernormal and Supernormal DVMs for Polyatomic Molecules}

We will below use the concept of "linearly independent" collisions [10]. Intuitively, a set of collisions is linearly dependent if one of them can be obtained by a combination of (some of) the other collisions (including corresponding reverse collisions), and correspondingly linearly independent if this is not the case. More formally, each collision can be represented by an $n$-dimensional vector with $0,-1$, and 1 as the only coordinates, see, e.g., $[16,18]$, in the way that collision (7) is represented by a vector (with non-zero elements at the positions $i, j, k$, and $l$ )

$$
(0, \ldots, 0, \underbrace{1}_{i}, 0, \ldots, 0, \underbrace{1}_{j}, 0, \ldots, 0, \underbrace{-1}_{k}, 0, \ldots, 0, \underbrace{-1}_{l}, 0, \ldots, 0) \in \mathbb{Z}^{n} .
$$

We then say that a set of collisions is linearly independent if and only if the set of the corresponding vectors is linearly independent. Furthermore, all vectors for possible collisions, i.e., collisions such that the collision coefficient $\Gamma_{i j}^{k l}(3)$ is nonzero, $\Gamma_{i j}^{k l} \neq 0$, can be put as rows in a matrix of collisions $\Lambda$ (in fact, it is enough that the vectors for all linearly independent collisions are included) $[16,18]$. Then the kernel of $\Lambda$ will be equal to the vector space of collisions invariants $[16,18]$, and hence, the number of collision invariants is given by

$$
\operatorname{dim}(\operatorname{ker}(\Lambda))=n-\operatorname{rank}(\Lambda) .
$$

Therefore, for a normal DVM we need to have $[16,18]$

$$
\operatorname{rank}(\Lambda)=n-(d+2)
$$

Note that by the conditions on the collision coefficient $\Gamma_{i j}^{k l}(3)$, we will always have the trivial collision invariants (8), and hence,

$$
\operatorname{rank}(\Lambda) \leq n-(d+2)
$$

We will now present a possible strategy for constructing (semi-)supernormal DVMs for polyatomic molecules.

\section{Algorithm for construction of semi-supernormal DVMs for polyatomic molecules}

(1) Choose a set of velocities $V_{1}$ such that it corresponds to a normal DVM for a monatomic species. Here, and in all the steps below, the set should be chosen in such a way, that we can obtain normal models for any mass ratio and/or energy levels we intend to consider; otherwise we might also be able to extend the set(s) later, as we realize that it is needed.

(2) Iteration step. For $j=2, \ldots, s$ :

Choose a set of velocities $\mathrm{V}_{j}$ [in a similar way as in step (1)] such that, there is $1 \leq i<j$, such that

$$
\left\{\left\{\mathrm{V}_{i}, E^{i}\right\},\left\{\mathrm{V}_{j}, E^{j}\right\}\right\}
$$

is a normal DVM. 
Remark 1 If we do not allow any collisions between the two levels of internal energies, we will have exactly $2 d+4$ linearly independent collision invariants, since, if $n_{i}$ and $n_{j}$ are the number of velocities in $\mathrm{V}_{i}$ and $\mathrm{V}_{j}$, respectively, the rank of the matrix of collisions $\Lambda$ will be

$$
\operatorname{rank}(\Lambda)=n_{i}-(d+2)+n_{j}-(d+2)=n_{i j}-(2 d+4), \quad n_{i j}=n_{i}+n_{j} .
$$

By Definition 1, we would like to have $d+2$ linearly independent collision invariants. Hence, we need to have $d+2$ linearly independent collisions between the two levels of internal energies. Hence, if, as in the case of all the (explicitly and implicitly) constructed DVMs in Sect. 3 (but, also for many others), there are $d+1$ linearly independent elastic collisions, we will only need to find one "basal" inelastic collision, if we have a maximal number of linearly independent elastic collisions. In fact, then by inequality (13) there will be at most one linearly independent inelastic collision with respect to the elastic collisions. This will not at all mean that we do not have more inelastic collisions, since a certain number of other inelastic collisions can be obtained by combining the basal one with elastic collisions (collisions represented by linear combinations of the vectors of collisions of the basal inelastic collision and elastic collisions). However, the obtained inelastic collisions will be linearly dependent with the elastic collisions and the basal inelastic one.

Note that in the spirit of Remark 1 we will have satisfactorily many inelastic collisions as long as we have satisfactorily many elastic collisions.

\section{Algorithm for construction of supernormal DVMs for polyatomic molecules}

(1) Choose a set of velocities $V_{1}$ such that it corresponds to a normal DVM for a monatomic species. As above, here, and in all the steps below, the set should be chosen in such a way, that we can obtain normal models for any mass ratio and/or energy levels we intend to consider; otherwise we might also be able to extend the set(s) later, as we realize that it is needed.

(2) Iteration step. For $j=2, \ldots, s$ :

Choose a set of velocities $\mathrm{V}_{j}$ [in a similar way as in step (1)] such that

$$
\left\{\left\{\mathrm{V}_{i}, E^{i}\right\},\left\{\mathrm{V}_{j}, E^{j}\right\}\right\}
$$

is a normal DVM for each $1 \leq i<j$. Also here, Remark 1 is applicable, in all cases.

\section{Construction of a Family of Supernormal DVMs with Internal Energies}

This section concerns an approach of the construction of supernormal DVMs with internal energies. We will use an odd-integer grid as our basic universe, instead of the usual integer grid, since in some applications (e.g., boundary layers $[4-6,8,13]$ ) it is preferable that the first component of the velocity is non-zero. However, the integer grid and the odd-integer grid are the same up to a shift and a scaling, and, of course, the odd-integer grid is also contained in the integer grid. Hence, if we would like to, we could also use the integer grid as our basic universe. If desirable, it is also possible to find "larger" normal (and symmetric) DVMs that contains the velocity sets for all different energy levels and hence, can be used as a common velocity set for all energy levels. We are concerned with finding $d+2$ linearly independent (also with respect to the collisions inside the energy levels) collisions between 
each energy levels. These collisions are not the only ones between each two energy levels, but all collisions between each two energy levels can be obtained by combining (one or more of) those linearly independent collisions (including corresponding reverse collisions) with the collisions inside energy levels, cf. Remark 1 above.

We now give a concrete example of the construction of normal DVMs with internal energies $E^{r}=r E, r=1, \ldots, s$, for some positive number $E$. We start with a set of velocities $\mathrm{V}$, which includes the six velocities

$$
\{( \pm 1, \pm 1),(3, \pm 1)\}
$$

for $d=2$ or the ten velocities

$$
\{( \pm 1, \pm 1, \pm 1),(3, \pm 1,1)\}
$$

for $d=3$. The minimal models (only containing these velocities) are normal, but can easily be extended to larger normal models (of any finite size) by so-called one-extensions [14$16,35]$. The smallest "symmetric" normal models containing the minimal models are the 12-velocity model

$$
( \pm 1, \pm 1),( \pm 3, \pm 1),( \pm 1, \pm 3)
$$

for $d=2$, and for $d=3$ the 32-velocity model

$$
( \pm 1, \pm 1, \pm 1),( \pm 3, \pm 1, \pm 1),( \pm 1, \pm 3, \pm 1),( \pm 1, \pm 1, \pm 3) .
$$

We let

$$
\mathrm{V}_{i}=\frac{\sqrt{E}}{2 \sqrt{m}} \mathrm{~V}, \quad i=1, \ldots, s,
$$

where $m$ denotes the mass. More generally, we can use different sets V (as long as they contain the necessary velocities) for different internal energies. As noted above, the minimal models are normal DVMs. A drawback of the minimal models is that the maximal total change of energies under a collision is $E$, i.e., except the elastic collisions, only collisions such that

$$
E_{i}+E_{j}-E_{k}-E_{l}= \pm E
$$

[cf. Eq. (4)] are possible. However, by enlarging these models we can get more possibilities.

Lemma 1 Let $d=2$ or 3. For any two given internal energies $r E$ and $q E$, where $r$ and $q$ are positive integers, such that $r<q$, there is a supernormal DVM

$$
\left\{\left\{\mathrm{V}_{r}, r E\right\},\left\{\mathrm{V}_{q}, q E\right\}\right\} .
$$

Proof For $d=2$, let V be a normal DVM, such that

$$
\{( \pm 1, \pm 1),(3, \pm 1),(2(q-r)-1,1),(2(q-r)+1,1)\} \subseteq \mathrm{V} .
$$

Such normal DVMs can be obtained from the normal DVM (14) by the method of oneextensions. Here we remind that a one-extension is obtained by adding a new velocity, chosen in such a way that there is a possible (i.e., such that the physical quantities are conserved) collision involving the new velocity and already existing velocities, to a normal DVM; and hence, obtain a new normal DVM with one more velocity. Furthermore, let

$$
\mathrm{V}_{r}=\mathrm{V}_{q}=\frac{\sqrt{E}}{2 \sqrt{m}} \mathrm{~V},
$$


where $m$ denotes the mass. Without any collisions between the different species we will have, since the DVMs are normal, the collision invariants

$$
\phi=\left(\phi^{r}, \phi^{q}\right), \quad \phi_{j}^{\alpha}=a_{\alpha}+m \mathbf{b}^{\alpha} \cdot \xi_{j}^{\alpha}+c_{\alpha}\left(m\left|\xi_{j}^{\alpha}\right|^{2}+2 \alpha E\right), \quad 1 \leq j \leq n_{\alpha},
$$

with $a_{\alpha}, c_{\alpha} \in \mathbb{R}, \mathbf{b}^{\alpha}=\left(b_{1}^{\alpha}, b_{2}^{\alpha}\right) \in \mathbb{R}^{2}$, and $\alpha \in\{r, q\}$. The collisions obtained by

$$
\begin{aligned}
& \left\{\left(\frac{\sqrt{E}}{2 \sqrt{m}}(1,1), r E\right),\left(\frac{\sqrt{E}}{2 \sqrt{m}}(-1,1), q E\right)\right\} \\
& \leftrightarrows\left\{\left(\frac{\sqrt{E}}{2 \sqrt{m}}(-1,1), r E\right),\left(\frac{\sqrt{E}}{2 \sqrt{m}}(1,1), q E\right)\right\}, \\
& \left\{\left(\frac{\sqrt{E}}{2 \sqrt{m}}(1,1), r E\right),\left(\frac{\sqrt{E}}{2 \sqrt{m}}(1,-1), q E\right)\right\} \\
& \leftrightarrows\left\{\left(\frac{\sqrt{E}}{2 \sqrt{m}}(1,-1), r E\right),\left(\frac{\sqrt{E}}{2 \sqrt{m}}(1,1), q E\right)\right\},
\end{aligned}
$$

and

$$
\begin{aligned}
& \left\{\left(\frac{\sqrt{E}}{2 \sqrt{m}}(1,1), r E\right),\left\{\frac{\sqrt{E}}{2 \sqrt{m}}(1,3), q E\right\}\right\} \\
& \leftrightarrows\left\{\left(\frac{\sqrt{E}}{2 \sqrt{m}}(1,3), r E\right),\left(\frac{\sqrt{E}}{2 \sqrt{m}}(1,1), q E\right)\right\},
\end{aligned}
$$

respectively, will imply that $b_{1}^{r}=b_{1}^{q}, b_{2}^{r}=b_{2}^{q}$, and $c_{r}=c_{q}$, respectively. Furthermore, the collisions obtained by

$$
\begin{aligned}
& \left\{\left\{\frac{\sqrt{E}}{2 \sqrt{m}}(2(q-r)+1,1), r E\right\},\left\{\frac{\sqrt{E}}{2 \sqrt{m}}(-1,-1), r E\right\}\right\} \\
& \leftrightarrows\left\{\left\{\frac{\sqrt{E}}{2 \sqrt{m}}(2(q-r)-1,1), r E\right\},\left\{\frac{\sqrt{E}}{2 \sqrt{m}}(1,-1), q E\right\}\right\}
\end{aligned}
$$

will imply that $a_{r}=a_{q}$.

For $d=3$, let $\mathrm{V}$ be a normal DVM, such that

$$
\{( \pm 1, \pm 1, \pm 1),(3, \pm 1,1),(2(q-r)-1,1),(2(q-r)+1,1)\} \subseteq \mathrm{V} .
$$

Such normal DVMs can be obtained from the normal DVM (15) by the method of oneextensions. As for the case $d=2$, let

$$
\mathrm{V}_{r}=\mathrm{V}_{q}=\frac{\sqrt{E}}{2 \sqrt{m}} \mathrm{~V} .
$$

Without any collisions between the different species we will have, since the DVMs are normal, the collision invariants

$$
\phi=\left(\phi^{r}, \phi^{q}\right), \quad \text { where } \phi_{j}^{\alpha}=a_{\alpha}+m \mathbf{b}^{\alpha} \cdot \xi_{j}^{\alpha}+c_{\alpha}\left(m\left|\xi_{j}^{\alpha}\right|^{2}+2 \alpha E\right) \quad \text { for } 1 \leq j \leq n_{\alpha},
$$


with $a_{\alpha}, c_{\alpha} \in \mathbb{R}, \mathbf{b}^{\alpha}=\left(b_{1}^{\alpha}, b_{2}^{\alpha}, b_{3}^{\alpha}\right) \in \mathbb{R}^{3}$, and $\alpha \in\{r, q\}$. The collisions obtained by

$$
\begin{aligned}
& \left\{\left(\frac{\sqrt{E}}{2 \sqrt{m}}(1,1,1), r E\right),\left(\frac{\sqrt{E}}{2 \sqrt{m}}(-1,1,1), q E\right)\right\} \\
& \leftrightarrows\left\{\left(\frac{\sqrt{E}}{2 \sqrt{m}}(-1,1,1), r E\right),\left(\frac{\sqrt{E}}{2 \sqrt{m}}(1,1,1), q E\right)\right\}, \\
& \left\{\left(\frac{\sqrt{E}}{2 \sqrt{m}}(1,1,1), r E\right),\left(\frac{\sqrt{E}}{2 \sqrt{m}}(1,-1,1), q E\right)\right\} \\
& \leftrightarrows\left\{\left(\frac{\sqrt{E}}{2 \sqrt{m}}(1,-1,1), r E\right),\left(\frac{\sqrt{E}}{2 \sqrt{m}}(1,1,1), q E\right)\right\}, \\
& \left\{\left(\frac{\sqrt{E}}{2 \sqrt{m}}(1,1,1), r E\right),\left(\frac{\sqrt{E}}{2 \sqrt{m}}(1,1,-1), q E\right)\right\} \\
& \leftrightarrows\left\{\left(\frac{\sqrt{E}}{2 \sqrt{m}}(1,1,-1), r E\right),\left(\frac{\sqrt{E}}{2 \sqrt{m}}(1,1,1), q E\right)\right\},
\end{aligned}
$$

and

$$
\begin{aligned}
& \left\{\left(\frac{\sqrt{E}}{2 \sqrt{m}}(1,1,1), r E\right),\left(\frac{\sqrt{E}}{2 \sqrt{m}}(1,3,1), q E\right)\right\} \\
& \leftrightarrows\left\{\left(\frac{\sqrt{E}}{2 \sqrt{m}}(1,3,1), r E\right),\left(\frac{\sqrt{E}}{2 \sqrt{m}}(1,1,1),\right)\right\},
\end{aligned}
$$

respectively, will imply that $b_{1}^{r}=b_{1}^{q}, b_{2}^{r}=b_{2}^{q}, b_{3}^{r}=b_{3}^{q}$, and $c_{r}=c_{q}$, respectively. Furthermore, the collisions obtained by

$$
\begin{aligned}
& \left\{\left(\frac{\sqrt{E}}{2 \sqrt{m}}(2(q-r)+1,1,1), r E\right),\left(\frac{\sqrt{E}}{2 \sqrt{m}}(-1,-1,1), r E\right)\right\} \\
& \leftrightarrows\left\{\left(\frac{\sqrt{E}}{2 \sqrt{m}}(2(q-r)-1,1,1), r E\right),\left(\frac{\sqrt{E}}{2 \sqrt{m}}(1,-1,1), q E\right)\right\}
\end{aligned}
$$

will imply that $a_{r}=a_{q}$.

Note that the sets of velocities used in the proofs of Lemma 1, in no way are unique. Furthermore, there can be sets of velocities that do not contain the velocities assumed in the proof, but still are supernormal for the given internal energies. We have just proven that there exist such sets of velocities for any given two internal energies $r E$ and $q E$, where $r$ and $q$ are positive integers.

Example 1 Assume that $d=2, s=2, r=1$, and $q=2$, with internal energies $E$ and $2 E$, and let

$$
\mathrm{V}=\{( \pm 1, \pm 1),(3, \pm 1)\},
$$

which is a normal DVM, in Eq. (16). Then the collisions (17)-(19) are represented by the blue/dotted $(. . \cdots)$ rectangles in Fig. 1, while the red/broken $\left(-{ }_{-}\right)$triangle represents the collision (20). The green/solid line squares represent a full set of linearly independent 
Fig. 1 12-Velocity supernormal model with internal energies $\mathrm{E}$ and $2 \mathrm{E}$

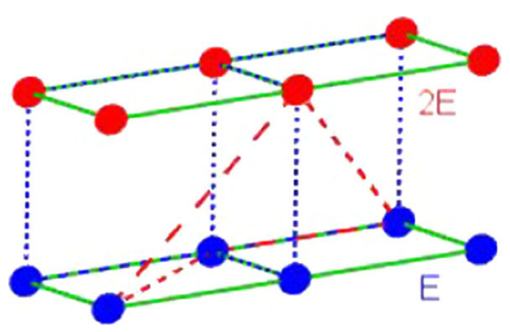

collisions for the DVMs of each internal energy level. All inelastic collisions, such that $\left\{E_{i}, E_{j}\right\} \neq\left\{E_{k}, E_{l}\right\}$, are given by the 16 collisions (all reversions included):

$$
\begin{aligned}
& \left\{\left(\frac{\sqrt{E}}{2 \sqrt{m}}(-1, \pm 1), E\right),\left(\frac{\sqrt{E}}{2 \sqrt{m}}(3, \mp 1), E\right)\right\} \\
& \leftrightarrows\left\{\left(\frac{\sqrt{E}}{2 \sqrt{m}}(1,-1), E / 2 E\right),\left(\frac{\sqrt{E}}{2 \sqrt{m}}(1,1), 2 E / E\right)\right\}, \\
& \left\{\left(\frac{\sqrt{E}}{2 \sqrt{m}}(-1, \pm 1), E / 2 E\right),\left(\frac{\sqrt{E}}{2 \sqrt{m}}(3, \mp 1), 2 E / E\right)\right\} \\
& \leftrightarrows\left\{\left(\frac{\sqrt{E}}{2 \sqrt{m}}(1,-1), 2 E\right),\left(\frac{\sqrt{E}}{2 \sqrt{m}}(1,1), 2 E\right)\right\} .
\end{aligned}
$$

Example 2 We now consider the case $d=2$ and $s=3$, with internal energies $E, 2 E$, and $4 E$. If we let

$$
\mathrm{V}=\{( \pm 1, \pm 1),(3, \pm 1),(1, \pm 3),(3,3)\},
$$

in Eq. (16), then we obtain a semi-supernormal DVM (see Fig. 2). On the other hand, if we let

$$
\mathrm{V}=\{( \pm 1, \pm 1),( \pm 1,-3),(3, \pm 1),(1,3),(3,3)\}
$$

in Eq. (16), then we obtain a supernormal DVM (see Fig. 3). Instead of using the same V for all levels of internal energy, we can use different sets for different levels of internal energy. In Fig. 4, the DVM is still supernormal, even if we have reduced some of the used sets. In Fig. 2 the collisions (17)-(19) are represented by the blue/dotted $(\cdots \cdots)$ rectangles and the collision (20) (for $\{r, q\}=\{1,2\}$ and $\{r, q\}=\{2,4\}$ ) by the red/broken $\left(-{ }_{-}\right.$) triangles. However, the collision (20) is missing for $\{r, q\}=\{1,4\}$, and so the DVM fails to be supernormal. However, in Figs. 3 and 4 the collision (20) for $\{r, q\}=\{1,4\}$ is represented by the brown/chain lines ( _ _ _ - ), and so the DVMs are supernormal. Again, the green/solid line squares represent a full set of linearly independent collisions for the DVMs of each internal energy level.

Theorem 3 Let $d=2$ or $d=3$. For any given set of internal energies $\left\{r_{1} E, \ldots, r_{s} E\right\}$, where $r_{1}, \ldots, r_{s}$ are positive integers, there is a supernormal DVM

$$
\left\{\left\{\mathrm{V}_{r_{1}}, r_{1} E\right\}, \ldots,\left\{\mathrm{V}_{r_{s}}, r_{s} E\right\}\right\} .
$$

Proof This is an immediate consequence of Theorem 2 and Lemma 1. 
Fig. 2 27-Velocity

semi-supernormal model with internal energies $\mathrm{E}, 2 \mathrm{E}$, and $4 \mathrm{E}$
Fig. 3 30-Velocity supernormal model with internal energies $\mathrm{E}$, $2 \mathrm{E}$, and $4 \mathrm{E}$

Fig. 4 25-Velocity supernormal model with internal energies $\mathrm{E}$, $2 \mathrm{E}$, and $4 \mathrm{E}$
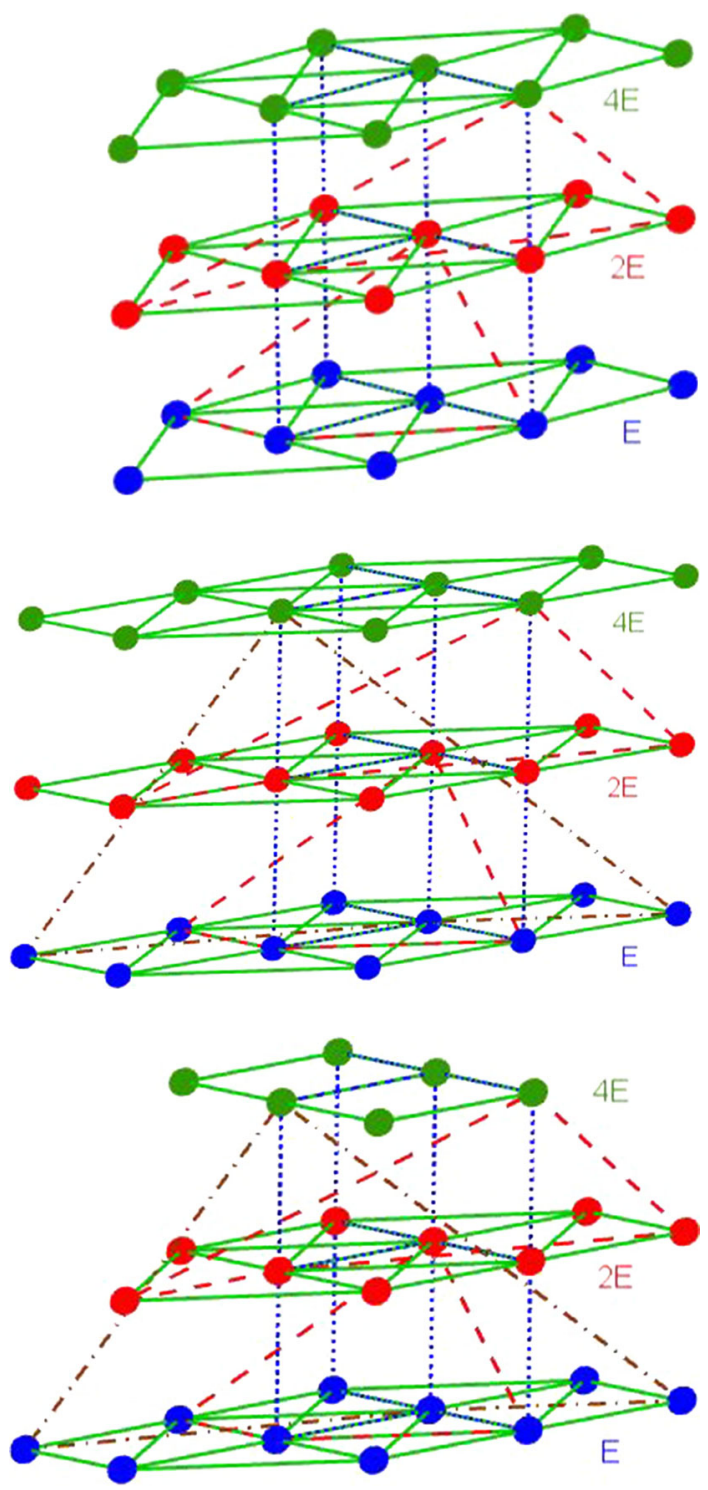

Remark 2 Lemma 1 and Theorem 3 can in an obvious way also be proved to be valid for any $d \geq 4$.

\section{DVMs for Mixtures with Polyatomic Molecules}

We can combine the approach for DVMs for single species with polyatomic molecules with the approach for DVMs for multicomponent mixtures [10] in an obvious way to obtain models for mixtures of polyatomic molecules [7]. We note that some (or even all) of the species actually can be monatomic. For that purpose assume that we have $s, s \geq 1$, different 
species, labelled with $\alpha_{1}, \ldots, \alpha_{s}$, with the masses $m_{\alpha_{1}}, \ldots, m_{\alpha_{s}}$, and that we for each species $\alpha_{i}$ have $r_{i}, r_{i} \geq 1$, different internal energies $E_{i}^{1}, \ldots, E_{i}^{r_{i}}$. Note that $s=r_{1}=1$ will give us the case of single species of monatomic molecules, $s>1$ with $r_{1}=\cdots=r_{s}=1$ will generate mixtures of monatomic molecules [10], while $s=1$ will give us the case of single species of polyatomic molecules discussed above.

For each species $\alpha_{i}$ and internal energy $E_{i}^{j}$ we fix a set of velocity vectors $\mathrm{V}_{i}^{j}=$ $\left\{\xi_{1}^{i j}, \ldots, \xi_{n_{i j}}^{i j}\right\} \subset \mathbb{R}^{d}$ and assign the label $\alpha_{i}$ and the internal energy $E_{i}^{j}$ to each velocity vector in $\mathrm{V}_{i}^{j}$. There will be $n=\sum_{i=1}^{s} \sum_{j=1}^{r_{i}} n_{i j}$ different triples, composed of a velocity vector, a label, and an internal energy, in the set

$$
\begin{aligned}
\mathscr{V} & =\left\{\left(\xi_{1}^{1,1}, \alpha_{1}, E_{1}^{1}\right), \ldots,\left(\xi_{n_{1,1}, 1}^{1}, \alpha_{1}, E_{1}^{1}\right), \ldots,\left(\xi_{1}^{s r_{s}}, \alpha_{s}, E_{s}^{r_{s}}\right), \ldots,\left(\xi_{n_{s r_{s}}}^{s r_{s}}, \alpha_{s}, E_{s}^{r_{s}}\right)\right\} \\
& =\left\{\left(\mathbf{v}_{1}, \alpha(1), E_{1}\right), \ldots,\left(\mathbf{v}_{n}, \alpha(n), E_{n}\right)\right\} .
\end{aligned}
$$

Note that the same velocity can be repeated many times, but only for different species and/or internal energies, since the triples are unique. Again we may need to scale the distribution functions, cf. Eq. (6), to be able to obtain the symmetry relations (3) for the collision coefficients (assuming a convenient reciprocity relation [25, p. 9])

$$
f_{r}^{\prime}=\frac{f_{r}}{g_{i}^{j}} \text { if } E_{r}=E_{i}^{j}, \quad r=1, \ldots, n \text {, for some numbers } g_{i}^{j}, \quad 1 \leq i \leq s, \quad 1 \leq j \leq r_{i} .
$$

Now $f_{i}=f_{i}(\mathbf{x}, t)=f\left(\mathbf{x}, t, \mathbf{v}_{i}, \alpha(i), E_{i}\right)$ represents the microscopic density of particles [of species $\alpha(i)$ and with internal energy $E_{i}$ ] with velocity $\mathbf{v}_{i}$ at time $t \in \mathbb{R}_{+}$and position $\mathbf{x} \in \mathbb{R}^{d}$ in Eq. (1). Note that, if we have made the scaling (21) then the left-hand side in Eq. (1) will be multiplied with the different scaling factors $g_{i}^{j}$. However, since here the scaling factors will have no significant effect on the structure of the results, we will for brevity leave them out [as in Eq. (1)].

The collision coefficients (3) in Eqs. (1)-(2) are now equal to zero unless we have conservation of mass for each species, momentum, and total energy:

$$
\begin{aligned}
\{\alpha(i), \alpha(j)\} & =\{\alpha(k), \alpha(l)\}, \\
m_{\alpha(i)} \mathbf{v}_{i}+m_{\alpha(j)} \mathbf{v}_{j} & =m_{\alpha(k)} \mathbf{v}_{k}+m_{\alpha(l)} \mathbf{v}_{l}, \\
\frac{m_{\alpha(i)}\left|\mathbf{v}_{i}\right|^{2}}{2}+\frac{m_{\alpha(j)}\left|\mathbf{v}_{j}\right|^{2}}{2}+E_{i}+E_{j} & =\frac{m_{\alpha(k)}\left|\mathbf{v}_{k}\right|^{2}}{2}+\frac{m_{\alpha(l)}\left|\mathbf{v}_{l}\right|^{2}}{2}+E_{k}+E_{l} .
\end{aligned}
$$

A collision is obtained by the exchange of velocities and/or internal energies

$$
\left\{\left(\mathbf{v}_{i}, \alpha(i), E_{i}\right),\left(\mathbf{v}_{j}, \alpha(j), E_{j}\right)\right\} \leftrightarrows\left\{\left(\mathbf{v}_{k}, \alpha(k), E_{k}\right),\left(\mathbf{v}_{l}, \alpha(l), E_{l}\right)\right\}
$$

and can occur if and only if $\Gamma_{i j}^{k l} \neq 0$. Geometrically, a collision obtained by (22) is represented by an isosceles trapezoid in $\mathbb{R}^{d}$, if $\left\{E_{i}, E_{j}\right\}=\left\{E_{k}, E_{l}\right\}$ [a rectangle if additionally $\alpha(i)=$ $\alpha(j)$, or more generally if and only if $\left.m_{\alpha(i)}=m_{\alpha(j)}\right]$, with the corners in $\left\{\mathbf{v}_{i}, \mathbf{v}_{j}, \mathbf{v}_{k}, \mathbf{v}_{l}\right\}$, where $\mathbf{v}_{i}$ and $\mathbf{v}_{j}$ (and hence, also $\mathbf{v}_{k}$ and $\mathbf{v}_{l}$ ) are diagonal corners, and

$$
m_{\alpha(i)}\left|\mathbf{v}_{i}-\mathbf{v}_{k}\right|=m_{\alpha(j)}\left|\mathbf{v}_{j}-\mathbf{v}_{l}\right|
$$

if $\alpha(i)=\alpha(k)$; with $k$ and $l$ interchanged in (23) otherwise, i.e., if $\alpha(i)=\alpha(l)$. In general, the collision obtained by (22) is geometrically represented by a trapezoid in $\mathbb{R}^{d}$ [a parallelogram if additionally $\alpha(i)=\alpha(j)$, or more generally if and only if $m_{\alpha(i)}=m_{\alpha(j)}$, with the corners 
in $\left\{\mathbf{v}_{i}, \mathbf{v}_{j}, \mathbf{v}_{k}, \mathbf{v}_{l}\right\}$, where $\mathbf{v}_{i}$ and $\mathbf{v}_{j}$ (and therefore, also $\mathbf{v}_{k}$ and $\mathbf{v}_{l}$ ) are diagonal corners, such that Eq. (23) and

$$
m_{\alpha(i)}\left(\mathbf{v}_{k}-\mathbf{v}_{i}\right) \cdot\left(\mathbf{v}_{l}-\mathbf{v}_{i}+\mathbf{v}_{j}-\mathbf{v}_{k}\right)=2\left(E_{k}+E_{l}-E_{i}-E_{j}\right)
$$

are fulfilled, if $\alpha(i)=\alpha(k)$; with $k$ and $l$ interchanged in Eqs. (23) and (24) otherwise, i.e., if $\alpha(i)=\alpha(l)$.

The trivial collision invariants (or physical collision invariants) are now

$$
1_{\alpha_{1}}, \ldots, 1_{\alpha_{s}}, m v^{1}, \ldots, m v^{d}, m|\mathbf{v}|^{2}+2 E
$$

(including all possible linear combinations), where $m=m(\mathbf{v})=\left(m_{\alpha(1)}, \ldots, m_{\alpha(n)}\right)$, $v^{i}=\left(v_{1}^{i}, \ldots, v_{n}^{i}\right)\left[\right.$ with $\left.\mathbf{v}_{j}=\left(v_{j}^{1}, \ldots, v_{j}^{d}\right)\right],|\mathbf{v}|^{2}=\left(\left|\mathbf{v}_{1}\right|^{2}, \ldots,\left|\mathbf{v}_{n}\right|^{2}\right), E=E(\mathbf{v})=$ $\left(E_{1}, \ldots, E_{n}\right)$, and

$$
1_{\alpha_{j}}=\left(\left(1_{\alpha_{j}}\right)_{1}, \ldots,\left(1_{\alpha_{j}}\right)_{n}\right), \quad\left(1_{\alpha_{j}}\right)_{i}=\delta_{\alpha_{j} \alpha(i)}=\left\{\begin{array}{l}
1 \text { if } \alpha(i)=\alpha_{j} \\
0 \text { if } \alpha(i) \neq \alpha_{j}
\end{array}\right.
$$

Normal DVMs are DVMs such that the set of the $s+d+1$ collision invariants (25) is a basis for the vector space of all collision invariants, i.e., all collision invariants are of the form

$$
\phi=\left(\phi_{1}, \ldots, \phi_{n}\right), \quad \phi_{i}=a_{\alpha(i)}+m_{\alpha(i)} \mathbf{b} \cdot \mathbf{v}_{i}+c\left(m_{\alpha(i)}\left|\mathbf{v}_{i}\right|^{2}+2 E_{i}\right)
$$

for some constant $a_{\alpha_{1}}, \ldots, a_{\alpha_{s}}, c \in \mathbb{R}$ and $\mathbf{b} \in \mathbb{R}^{d}$, and the stationary points or the Maxwellians are of the form (10), where for normal DVMs $\phi$ is now given by Eq. (26).

\subsection{Normal DVMs for Mixtures with Polyatomic Molecules}

In correspondence with the definitions in Sect. 2.1 we can introduce the following definitions.

\section{Definition 4 A DVM}

$$
\mathscr{V}=\left\{\left\{\mathrm{V}_{1}^{1}, \alpha_{1}, E_{1}^{1}\right\}, \ldots,\left\{\mathrm{V}_{1}^{r_{1}}, \alpha_{1}, E_{1}^{r_{1}}\right\}, \ldots,\left\{\mathrm{V}_{s}^{1}, \alpha_{s}, E_{s}^{1}\right\}, \ldots,\left\{\mathrm{V}_{s}^{r_{s}}, \alpha_{s}, E_{s}^{r_{s}}\right\}\right\},
$$

with internal energies $\left\{E_{i}^{j}: 1 \leq i \leq s, 1 \leq j \leq r_{i}\right\}$, is called normal if it is non-degenerate and has exactly $s+d+1$ linearly independent collision invariants [which by our assumptions will be of the form (26)].

Definition 5 A DVM (27) is called semi-supernormal if it is normal and the restriction to each velocity set $\mathrm{V}_{i}^{j}, 1 \leq i \leq s, 1 \leq j \leq r_{i}$, is a normal DVM.

Definition 6 A DVM (27) is called supernormal if the restriction to each non-empty subset of $\mathscr{V}$ constitutes a normal DVM.

In a natural way we can extend Theorem 2 [7] (see also corresponding theorem for mixtures in [10]).

Theorem 4 A DVM (27) is supernormal if and only if the restriction to each pair of sets in $\mathscr{V}$ constitutes a supernormal DVM.

Moreover, by combining the arguments in the proofs of the corresponding result for the particular cases of single species with polyatomic molecules (with $s=1$ ) in Theorem 3 and mixtures of monatomic molecules (with $r_{1}=\cdots=r_{s}=1$ ) in [10] 
Theorem 5 For any given number $s$ of species with given rational masses $m_{\alpha_{1}}, \ldots, m_{\alpha_{s}}$, and with $r_{i}$ given internal energies $\left\{r_{i 1} E, \ldots, r_{i r_{i}} E\right\}$ for a fixed $E \in \mathbb{R}_{+}$and rational numbers $r_{i 1}, \ldots, r_{i r_{i}}, i=1, \ldots, s$, there is a supernormal $D V M$ for the mixture of polyatomic molecules.

\subsection{Algorithm for Construction of Supernormal DVMs}

This section concerns an algorithm for for construction of supernormal DVMs for mixtures with polyatomic molecules.

Algorithm for construction of supernormal DVMs

(1) (a) Choose a set of velocities $V_{1}^{1}$ such that it corresponds to a normal DVM for a monatomic single species. Here, and in all the steps below, the set should be chosen in such a way, that we can obtain normal models for any mass ratio and/or energy levels we intend to consider; otherwise we might also be able to extend the set(s) later, as we realize that it is needed.

(b) For $j=2, \ldots, r_{1}$ : choose a set of velocities $\mathrm{V}_{1}^{j}$ corresponding to a normal DVM such that $\left\{\left\{\mathrm{V}_{1}^{k}, E_{1}^{k}\right\},\left\{\mathrm{V}_{1}^{j}, E_{1}^{j}\right\}\right\}$ is a normal DVM for each $1 \leq k<j$.

(2) For $i=2, \ldots, s$ :

(a) Choose a normal set of velocities $V_{i}^{1}$ such that it, together with each of $\mathrm{V}_{1}^{1}, \ldots, \mathrm{V}_{1}^{r_{1}}, \ldots, \mathrm{V}_{i-1}^{1}, \ldots, \mathrm{V}_{i-1}^{r_{i-1}}$, corresponds to a supernormal DVM for binary mixtures.

(b) For $j=2, \ldots, r_{i}$ : choose a set of velocities $\mathrm{V}_{i}^{j}$ such that

(i) $\mathrm{V}_{i}^{1}$ together with each of $\mathrm{V}_{1}^{1}, \ldots, \mathrm{V}_{1}^{r_{1}}, \ldots, \mathrm{V}_{i-1}^{1}, \ldots, \mathrm{V}_{i-1}^{r_{i-1}}$ corresponds to a supernormal DVM for binary mixtures;

(ii) $\left\{\left\{\mathrm{V}_{i}^{k}, E_{i}^{k}\right\},\left\{\mathrm{V}_{i}^{j}, E_{i}^{j}\right\}\right\}$ is a normal DVM for each $1 \leq k<j$.

We remind, see Sect. 2.2, that we call a set of collisions for linearly dependent if one of them can be obtained by a combination of (some of) the other collisions (including corresponding inverse collisions), and correspondingly linearly independent otherwise.

Remark 3 In each case, if we do not allow any collisions between the two species/levels of internal energies, we will have $2 d+4$ linearly independent collision invariants, but we would like to have $d+3 / d+2$ linearly independent collision invariants for mixtures/polyatomic molecules respectively. Hence, cf. [16], we need to have $d+1 / d+2$ linearly independent (also with respect to the collisions inside the two species/energy levels) collisions between the two species/energy levels, cf. Remark 1.

In the case of explicit constructions we could, as in Sect. 3, use an odd-integer grid as our basic universe, instead of the usual integer grid (by the same arguments as in Sect. 3) and use the same base models as in Sect. 3 . Here we are concerned by finding $d+1 / d+2$ linearly independent (also with respect to the collisions inside the two species/energy levels) collisions between each two species/energy levels. Again, we stress that those collisions are not the only ones between each two species/energy levels, but all collisions between each species/energy levels can be obtained by combining (one or more of) those linearly independent collisions (including corresponding reverse collisions) with the collisions inside the species/energy levels.

Here we could let

$$
\mathrm{V}_{i}^{j}=\frac{\sqrt{E}}{2 m_{\alpha_{i}}} \mathrm{~V}, \quad i=1, \ldots, s .
$$




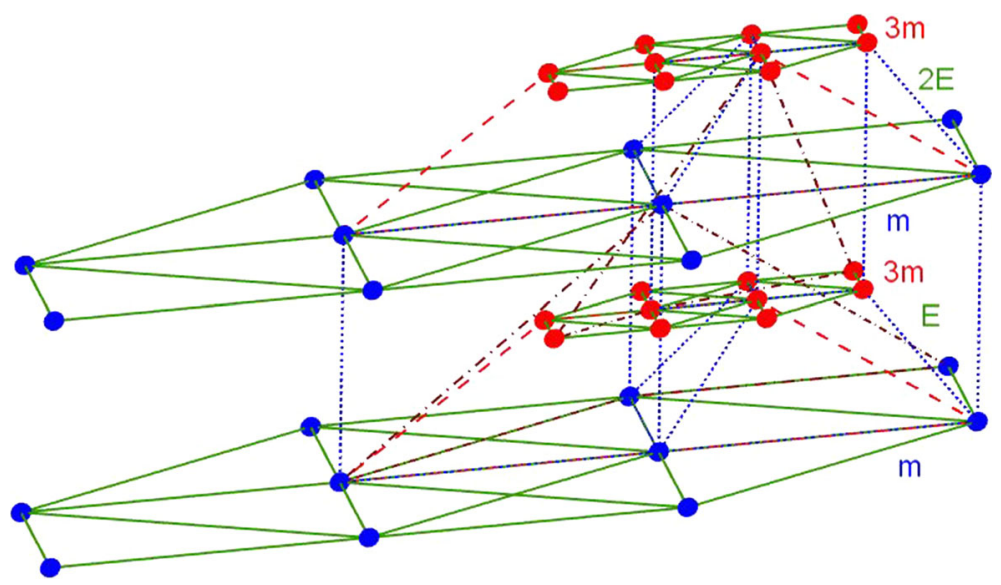

Fig. 5 40-Velocity supernormal model for a binary mixture with mass ratio 3 of polyatomic molecules with two internal energy levels $E$ and $2 E$

Again, instead of using the same set $\mathrm{V}$ for all species, we can also use different subsets of $\mathrm{V}$ for different species or vice versa extend the velocity set to be the same for all species and internal energies. Some explicit examples of such constructions can be found in [7], but we will also give some examples below.

Example 3 We consider, for brevity, the case $d=2$, and assume that $s=2$ and $r_{1}=r_{2}=2$, with the masses $m$ and $3 m$, and the internal energies $E$ and $2 E$. We denote

$$
\mathrm{V}=\{( \pm 1, \pm 1),(3, \pm 1),(1,3),(3,3),(5,1),(5,3)\},
$$

which constitutes a normal DVM. Then

$$
\mathrm{V}_{1}^{1}=\mathrm{V}_{1}^{2}=\frac{3 \sqrt{E}}{2 m} \mathrm{~V} ; \quad \mathrm{V}_{2}^{1}=\mathrm{V}_{2}^{2}=\frac{\sqrt{E}}{2 m} \mathrm{~V},
$$

constitutes a supernormal DVM, see Fig. 5. Instead of using the same V for different species and internal energy levels, we can use different velocity sets. In Fig. 6, the DVM is still supernormal, even if we have reduced the velocity set $\mathrm{V}$ to

$$
\{( \pm 1, \pm 1),(3, \pm 1))\}
$$

for the light species, and

$$
\{( \pm 1,1),(1,-1),(3, \pm 1),(1,3),(3,3),(5,1),\}
$$

for the heavy species at the higher internal energy level. However, the same velocity sets could also be used for a binary mixture with mass ratio 3 of polyatomic molecules with two internal energy levels $E$ and $2 E$ (heavy species), and $E$ and $4 E / 3$ (light species), if the (brown/chained) "basal" inelastic collision is changed, see Fig. 7 [where also the velocity set $\mathrm{V}$ for the heavy species at the lower internal energy level is reduced to (28)].

\subsection{Bimolecular Chemical Reactions}

We can also add bimolecular reactive collisions [11] (by changing corresponding collision coefficients to be nonzero) to DVMs for mixtures of polyatomic molecules and by that extend 


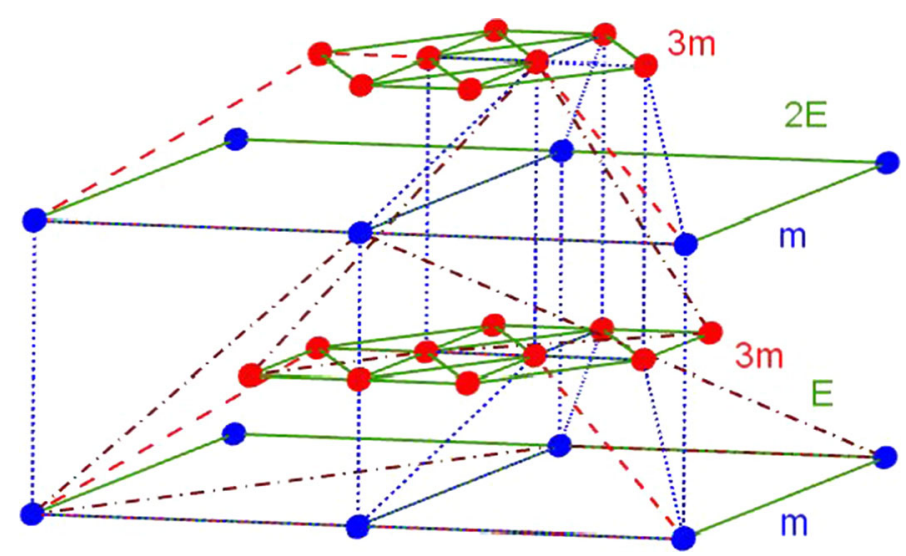

Fig. 630 -Velocity supernormal model for a binary mixture with mass ratio 3 of polyatomic molecules with two internal energy levels $E$ and $2 E$

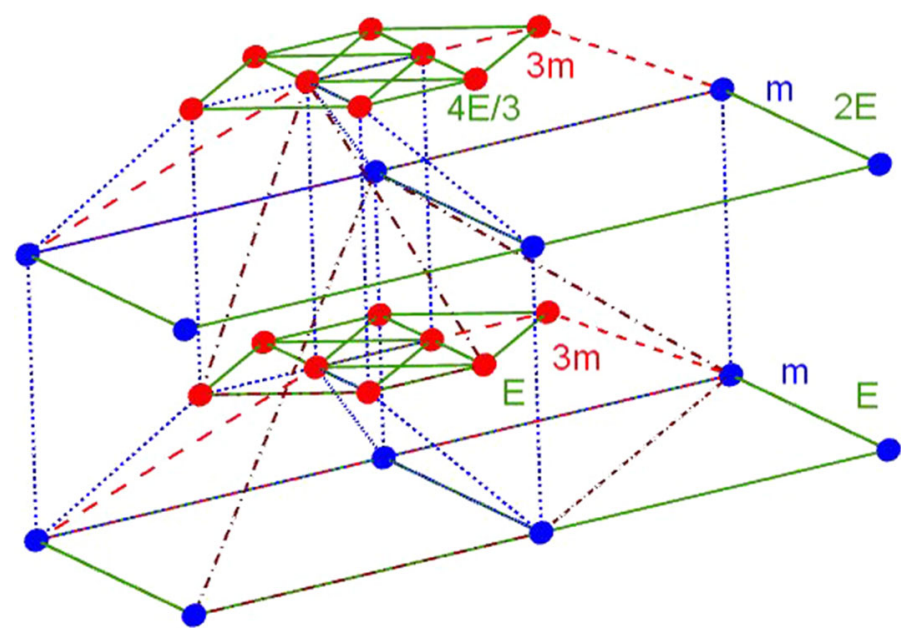

Fig. 7 28-Velocity supernormal model for a binary mixture with mass ratio 3 of polyatomic molecules with two internal energy levels $E$ and $2 E$ (heavy species), and $E$ and $4 E / 3$ (light species)

to DVMs for bimolecular chemical reactions. For each linearly independent (also with respect to all other collisions) reactive collision, we obtain one new relation on the masses. Note that the maximal number of linearly independent bimolecular reactive collisions are $d-1$, since the total number of particles will still be conserved. In [8] an example (cf. [29,33]) is considered. However, our method is not limited to that case, but can be used also in more general cases.

Acknowledgements The first ideas from which this paper originate were obtained during a visit at Parma University. The author wants to thank M. Groppi, G. Spiga, and M. Bisi at Parma University for valuable discussions.

Open Access This article is distributed under the terms of the Creative Commons Attribution 4.0 International License (http://creativecommons.org/licenses/by/4.0/), which permits unrestricted use, distribution, and 
reproduction in any medium, provided you give appropriate credit to the original author(s) and the source, provide a link to the Creative Commons license, and indicate if changes were made.

\section{References}

1. Baranger, C., Bisi, M., Brull, S., Desvillettes, L.: On the Chapman-Enskog asymptotics for a mixture of monatomic and polyatomic rarefied gases. Kinet. Relat. Models 11, 821-858 (2018)

2. Bardos, C., Caflisch, R.E., Nicolaenko, B.: The Milne and Kramers problems for the Boltzmann equation of a hard sphere gas. Commun. Pure Appl. Math. 39, 323-352 (1986)

3. Bardos, C., Golse, F., Sone, Y.: Half-space problems for the Boltzmann equation: a survey. J. Stat. Phys. 124, 275-300 (2006)

4. Bernhoff, N.: On half-space problems for the linearized discrete Boltzmann equation. Riv. Mat. Univ. Parma 9, 73-124 (2008)

5. Bernhoff, N.: On half-space problems for the weakly non-linear discrete Boltzmann equation. Kinet. Relat. Models 3, 195-222 (2010)

6. Bernhoff, N.: Boundary layers and shock profiles for the discrete Boltzmann equation for mixtures. Kinet. Relat. Models 5, 1-19 (2012)

7. Bernhoff, N.: Discrete velocity models for multicomponent mixtures and polyatomic molecules without nonphysical collision invariants and shock profiles. AIP Conf. Proc. 1786(1), 040005 (2016). https://doi. org/10.1063/1.4967543

8. Bernhoff, N.: Boundary layers for discrete kinetic models: multicomponent mixtures, polyatomic molecules, bimolecular reactions, and quantum kinetic equations. Kinet. Relat. Models 10, 925-955 (2017)

9. Bernhoff, N., Bobylev, A.: Weak shock waves for the general discrete velocity model of the Boltzmann equation. Commun. Math. Sci. 5, 815-832 (2007)

10. Bernhoff, N., Vinerean, M.C.: Discrete velocity models for multicomponent mixtures without nonphysical collision invariants. J. Stat. Phys. 165, 434-453 (2016)

11. Bird, G.A.: Molecular Gas Dynamics. Clarendon-Press, Oxford (1976)

12. Bisi, M., Spiga, G.: On kinetic models for polyatomic gases and their hydrodynamic limits. Ric. mat. 66, 113-124 (2017)

13. Bobylev, A.V., Bernhoff, N.: Discrete velocity models and dynamical systems. In: Bellomo, N., Gatignol, R. (eds.) Lecture Notes on the Discretization of the Boltzmann Equation, pp. 203-222. World Scientific, Singapore (2003)

14. Bobylev, A.V., Cercignani, C.: Discrete velocity models for mixtures. J. Stat. Phys. 91, 327-341 (1998)

15. Bobylev, A.V., Cercignani, C.: Discrete velocity models without non-physical invariants. J. Stat. Phys. 97, 677-686 (1999)

16. Bobylev, A.V., Vinerean, M.C.: Construction of discrete kinetic models with given invariants. J. Stat. Phys. 132, 153-170 (2008)

17. Bobylev, A.V., Palczewski, A., Schneider, J.: On approximation of the Boltzmann equation by discrete velocity models. C. R. Acad. Sci. Paris I 320, 639-644 (1995)

18. Bobylev, A.V., Vinerean, M.C., Windfall, A.: Discrete velocity models of the Boltzmann equation and conservation laws. Kinet. Relat. Models 3, 35-58 (2010)

19. Bourgat, J.F., Desvillettes, L., Le Tallec, P., Perthame, B.: Microreversible collisions for polyatomic gases and Boltzmann's theorem. Eur. J. Mech. B 13, 237-254 (1994)

20. Brechtken, S., Sasse, T.: Normal, high order discrete velocity models of the Boltzmann equation. Comput. Math. Appl. 75, 503-519 (2018)

21. Buet, C.: Conservative and entropy schemes for Boltzmann collision operator of polyatomic gases. Math. Models Methods Appl. Sci. 7, 165-192 (1997)

22. Caflisch, R.E., Nicolaenko, B.: Shock profile solutions of the Boltzmann equation. Commun. Math. Phys. 86, 161-194 (1982)

23. Cercignani, C., Cornille, H.: Shock waves for a discrete velocity gas mixture. J. Stat. Phys. 99, 115-140 (2000)

24. Cornille, H., Cercignani, C.: A class of planar discrete velocity models for gas mixtures. J. Stat. Phys. 99, 967-991 (2000)

25. Ern, A., Giovangigli, V.: Multicomponent Transport Algorithms. Springer, Berlin (1994)

26. Fainsilber, L., Kurlberg, P., Wennberg, B.: Lattice points on circles and discrete velocity models for the Boltzmann equation. SIAM J. Math. Anal. 37, 1903-1922 (2006)

27. Gatignol, R.: Théorie Cinétique des Gaz à Répartition Discrète de Vitesses. Springer, Berlin (1975) 
28. Goldstein, D.B.: Discrete-velocity collision dynamics for polyatomic molecules. Phys. Fluids A 4, 18311839 (1992)

29. Groppi, M., Spiga, G.: Kinetic approach to chemical reactions and inelastic transitions in a rarefied gas. J. Math. Chem. 26, 197-219 (1999)

30. Liu, T.P., Yu, S.H.: Boltzmann equation: micro-macro decompositions and positivity of shock profiles. Commun. Math. Phys. 246, 133-179 (2004)

31. Mouhot, C., Pareschi, L., Rey, T.: Convolutive decomposition and fast summation methods for discretevelocity approximations of the Boltzmann equation. Math. Model. Numer. Anal. 47, 1515-1531 (2013)

32. Palczewski, A., Schneider, J., Bobylev, A.V.: A consistency result for a discrete-velocity model of the Boltzmann equation. SIAM J. Numer. Anal. 34, 1865-1883 (1997)

33. Rossani, A., Spiga, G.: A note on the kinetic theory of chemically reacting gases. Physica A 272, 563-573 (1999)

34. Ukai, S., Yang, T., Yu, S.H.: Nonlinear boundary layers of the Boltzmann equation: I. Existence. Commun. Math. Phys. 236, 373-393 (2003)

35. Vedenyapin, V.V.: Velocity inductive method for mixtures. Transp. Theory Stat. Phys. 28, 727-742 (1999)

36. Vedenyapin, V.V., Amasov, S.A.: Discrete models of the Boltzmann equation for mixtures. Differ. Equ. 36, 1027-1032 (2000)

37. Vedenyapin, V.V., Orlov, Y.N.: Conservation laws for polynomial Hamiltonians and for discrete models of the Boltzmann equation. Theor. Math. Phys. 121, 1516-1523 (1999) 